\title{
DENSIDADE DE Rhea americana EM TRÊS PAISAGENS DIFERENTES DO PANTANAL DA NHECOLÂNDIA, MS
}

\author{
Diogo Maia Gräbin ,,, Marcelle Aiza Tomas² \& Walfrido Moraes Tomas ${ }^{2}$ \\ ${ }^{1}$ Universidade Federal do Rio Grande do Sul , Instituto de Biociências, Av. Bento Gonçalves, 9500, Campus do Vale, Bloco IV, Prédio 43433, Sala: \\ 207 - Porto Alegre, RS, Brasil CEP: 91501-970. \\ ${ }^{2}$ EMBRAPA Pantanal, Rua 21 de Setembro, 1880, Bairro Nossa Senhora de Fátima, Caixa Postal: 109, Corumbá, MS, Brasil. CEP:79320-900. \\ E-mails: diogo_grabin@yahoo.com.br,marcelletomas@gmail.com,walfrido.tomas@embrapa.br
}

\section{RESUMO}

Pecuária em campos nativos representa uma atividade econômica tradicional no Pantanal brasileiro. Contudo, a substituição da vegetação nativa por pastagens cultivadas de espécies exóticas tem aumentado. As respostas da fauna local em relação a essas transformações ainda são pouco conhecidas. Nosso objetivo foi estimar a densidade de ema (Rhea americana) em três diferentes paisagens para verificar possíveis respostas às suas diferenças. O estudo foi realizado em três fazendas do Pantanal da Nhecolândia, de maio a junho de 2010. Em cada fazenda foram percorridas de seis a sete transecções, repetidas vezes, entre 7:00h e 17:00h. Indivíduos ou grupos de emas avistados foram registrados, bem como as distâncias perpendiculares de cada grupo observado à linha da transecção. Os dados foram analisados utilizando o programa Distance 6.0. As densidades de ema foram estimadas em 1,086 $\pm 0,232$ indivíduos $/ \mathrm{km}^{2}$ em paisagem dominada por cerrado/ campo cerrado, 2,083 $\pm 0,440$ indivíduos $/ \mathrm{km}^{2}$ em paisagem com predominância de campo limpo e 2,030 \pm 0,411 indivíduos $/ \mathrm{km}^{2}$ na pastagem exótica cultivada. Os resultados indicam uma preferência das emas por áreas de campo aberto, o que sugere que esta espécie pode ser favorecida pela simplificação da paisagem em mosaico do Pantanal para a implantação de pastagens cultivadas.

Palavras-chave: amostragem de distâncias; ema; Rheidae; Urochloa spp.

\section{ABSTRACT}

DENSITY OF RHEA AMERICANA (RHEIDAE) IN THREE DIFFERENT LANDSCAPES IN THE

PANTANAL. Cattle ranching on native grasslands is a traditional economic activity in the Brazilian Pantanal. However, recently there is an increase in the replacement of the native vegetation by cultivated pastures with exotic species. The responses of the local fauna in relation to these changes are still poorly known. Our aim was to estimate the density of Greater Rhea (Rhea americana) in three landscapes in order to assess the response of population density to these differences. The study was conducted at three ranches located in the Nhecolândia region of the Pantanal, from May to June 2010. In each ranch, six to seven transects were surveyed repeatedly, from 7:00h to 17:00h. Sightings of individuals Rheas or groups were recorded as well as the perpendicular distance between the groups and the transect line. The data were analyzed using the software Distance 6.0. The Rhea density was estimated as $1.086 \pm 0.232$ individuals $/ \mathrm{km}^{2}$ at a landscape dominated by cerrado/campo cerrado, $2.083 \pm 0.440$ individuals $/ \mathrm{km}^{2}$ at grassland-dominated landscape, and $2.030 \pm 0.411$ individuals $/ \mathrm{km}^{2}$ at cultivated exotic pasture. These findings indicate a preference for open grassland landscapes by Greater Rheas, and suggests that this species may be favored by the simplification of the Pantanal's complex mosaic landscape due to the introduction cultivated pastures.

Keywords: distance sampling; greater Rhea; Rheidae; Urochloa spp.

\section{RESUMEN}

DENSIDAD EN TRES DIFERENTES RHEA AMERICANA ENPAISAJES PANTANAL DE LA NHECOLÂNDIA, MS. Ganadería en las praderas es una actividad económica tradicional en el Pantanal 
brasileño. Sin embargo, la sustitución de la vegetación nativa por pastos de especies exóticas se ha incrementado. Las respuestas de la fauna local para estas transformaciones son aún poco conocidos. Nuestro objetivo fue estimar la densidad de ñandú (Rhea americana) en tres paisajes diferentes para comprobar posibles respuestas a sus diferencias. El estudio se realizó en tres fincas de la Nhecolândia Pantanal, mayo-junio de 2010. En cada finca se cubrieron seis-siete transectos repetidamente 07:00-17:00. Los individuos o grupos de emúes avistados fueron grabadas y las distancias perpendiculares de cada grupo tomó nota de la línea de corte transversal. Los datos fueron analizados utilizando los 6,0 Distancia programa. Ema densidades se estimaron en 1,086 $\pm 0,232$ en individuos/km2 paisaje dominado por cerrado / cerrado y 2,083 $\pm 0,440$ en individuos/km2 paisaje con predominio de pastizales y 2,030 $\pm 0,411$ individuos $/ \mathrm{km} 2$ pasto cultivado exótico. Los resultados indican una preferencia por las áreas de emus campo abierto, lo que sugiere que esta especie puede verse favorecida por la simplificación del mosaico del paisaje del Pantanal para la implementación de pasturas.

Palabras clave: distancias de muestreo; ema; Rheidae; Urochloa spp.

\section{INTRODUÇÃO}

Populações de aves dependentes de habitats campestres têm declinado em vários locais ao redor do planeta, representando para alguns autores uma "crise proeminente para a conservação da vida silvestre no século 21" (Knopf 1994, Murphy 2003, Brennan \& Kuvlesky 2005). Em diversas situações os campos passaram a ser considerados ecossistemas ameaçados, bem como as espécies de aves que apresentam estreita relação com esses ambientes (Knopf 1994; Vickery et al. 1999, Bond \& Parr 2010). Dentre as principais ameaças aos ecossistemas campestres estão a fragmentação, a perda e a descaracterização do habitat por ação antrópica visando fins produtivos (Martin \& Finch 1995, Bond \& Parr 2010).

A ema, Rhea americana Linnaeus, 1758 (Struthioniformes, Rheidae) é a maior ave e uma das duas únicas espécies de ratitas na região neotropical (Azevedo et al. 2006, Erize et al. 2006). Diferentes subespécies distribuem-se pela Bolívia, Paraguai, Uruguai, Argentina e grande parte do Brasil, onde ocupa principalmente áreas de campo aberto e cerrado (Blake 1977, Erize et al. 2006) . Encontra-se quase ameaçada em nível global e, apesar de já extinta em alguns estados brasileiros, não é citada no Livro Vermelho da Fauna Brasileira Ameaçada de Extinção (Sick 1997, Silveira \& Straube 2008, BirdLife International 2012). No passado, a espécie sofreu forte pressão de caça em grande parte de sua área de ocorrência, visando aproveitamento de carne, pele e plumas, além da coleta de ovos (Sick 1997, BirdLife Internationa, 2012). Por ora, as populações de $R$. americana seguem declinando, mas principalmente em razão da supressão e descaracterização dos habitats (Bellis et al. 2004, BirdLife International 2012).

Uma das regiões onde $R$. americana ainda pode ser encontrada com relativa abundância é o Pantanal, a maior planície inundável contínua continental do planeta (Seidl et al. 2001, Tomas et al. 2004). A pecuária extensiva é realizada na planície pantaneira desde meados da metade do século 18, representando a principal atividade econômica da região, a qual utiliza a maior parte do uso da terra nessa região (Alho \& Lacher Jr. 1991, Seidl et al. 2001, Junk \& Cunha 2005, Alho \& Sabino 2011). Cerca de $95 \%$ das terras são privadas e $80 \%$ do Pantanal é usado para bovinocultura de manejo extensivo, no qual o gado é tradicionalmente mantido em baixas densidades e tem sua dieta baseada em pastagens nativas (Seidl et al. 2001, Junk \& Cunha 2005, Mercante et al. 2011). Contudo, nas últimas décadas, visando acompanhar a competitividade dos pecuaristas das áreas vizinhas ao Pantanal, os produtores locais têm procurado intensificar a produção investindo na substituição da vegetação natural por pastagens cultivadas, incrementando a nutrição dos rebanhos e amenizando a sazonal disponibilidade das pastagens nativas (Seidl et al. 2001, Padovani et al. 2004, Junk et al. 2006). Para isso, não somente campos nativos como também áreas florestais e savanas arbóreas estão sendo substituídas por gramíneas exóticas, o que vêm acontecendo num ritmo cada vez mais acelerado (Pott 1982, Padovani et al. 2004, Harris et al. 2005). As consequências dessas mudanças na composição 
e/ou estrutura das paisagens para a fauna local não são conhecidas para a maioria das espécies (Harris et al. 2005; Tizianel 2008), mas tem sido demonstrada para alguns grupos como quirópteros (Silveira 2011), aves (Tizianel 2008) e pequenos mamíferos (Antunes 2009, Mozerle 2011).

Dados de densidade populacional podem não só revelar quais habitats são os mais favoráveis para uma espécie como também são de fundamental importância para diagnosticar e monitorar sua situação em um dado local (Thompson et al. 1998). Esforços de conservação podem ser melhor direcionados a partir do entendimento de como a composição e estrutura da paisagem afetam as populações de espécies de aves campestres (Peterjohn 2003, Veech 2006, Bellis et al. 2008). Nosso objetivo foi estimar a densidade de $R$. americana em três paisagens diferentes para avaliar como a composição da paisagem e sua alteração afetam a abundância da espécie no Pantanal.

\section{MATERIAL E MÉTODOS}

O estudo foi realizado em três áreas adjacentes com distintos manejos voltados à bovinocultura, localizadas na sub-região do Pantanal da Nhecolândia, município de Corumbá, Mato Grosso do Sul, centrooeste do Brasil. A Nhecolândia está situada na porção sul do leque aluvial do rio Taquari, e se caracteriza por sua heterogeneidade ambiental composta por milhares de lagoas com características variadas, inclusive salinas, formações florestais nas áreas mais elevadas do terreno e formações campestres nas porções mais baixas, sazonalmente inundadas (Pott et al. 1986, Almeida et al. 2009) . Dentre as formações campestres distinguem-se a savana arborizada, conhecida como campo cerrado, e a savana gramíneo lenhosa (Santos et al. 2002). Na Fazenda Nhumirim (1859’21,0”S $\left.56^{\circ} 37^{\prime} 05,0^{\prime \prime} \mathrm{W}\right)$ foram amostradas áreas com predomínio de cerrado e campo cerrado nativo. $\mathrm{Na}$ Fazenda Ipanema (1903'54,0"S 56 34'28,0”W) foram amostradas áreas de pastagens exóticas cultivadas com diferentes espécies de braquiária (Urochloa sp.), caracterizadas por áreas campestres e áreas com árvores de grande porte deixadas isoladas no terreno mais alto, anteriormente ocupado por mata e cerradão. Estas pastagens foram implantadas em uma paisagem originalmente semelhante à da fazenda Nhumirim, com predominância de cerrado, da qual dista cerca de $5 \mathrm{~km}$. Já na Fazenda Alegria $\left(19^{\circ} 01^{\prime} 57,0\right.$ ' $\left.\mathrm{S} 56^{\circ} 46^{\prime} 36,0^{\prime \prime} \mathrm{W}\right)$ foram amostradas áreas naturais de campo limpo inundável e não inundável, caracterizadas pela predominância de campos entremeados por arbustos e pequenas manchas isoladas de habitats florestais, os capões.

Para estimar as densidades, utilizamos a amostragem de distâncias, devido ao seu baixo custo e à possibilidade de correção dos erros e diferenças na detectabilidade nas diferentes fisionomias (Cassey 1999, Buckland et al. 2001, Conroy \& Carrol 2009). O método da amostragem de distâncias é utilizado para encontrar a função que melhor descreve como a detectabilidade de uma espécie em um dado ambiente decai à medida que aumenta a distância perpendicular entre a linha da transeção e o indivíduo ou grupo de animais. No caso deste estudo, considerandose as premissas de que a densidade da espécie seja homogênea ao longo da área amostrada, que os animais sobre a linha da transeção ou próximos a ela são certamente percebidos pelo observador e baseando-se no comportamento dos dados observados e na função que a eles melhor se ajusta é possível então estimar quantos indivíduos passaram despercebidos durante os levantamentos em campo. Assim, torna-se possível estimar a densidade de grupos, quantos indivíduos são esperados por grupo e qual a densidade corrigida de indivíduos em uma dada área.

As transeções lineares foram definidas de forma que não passassem por outras fisionomias exceto a analisada, evitando-se áreas florestais. $\mathrm{O}$ comprimento dessas transeções variou de $1,6 \mathrm{~km}$ a $4,0 \mathrm{~km}$. No campo limpo nativo foram percorridas sete transeções, com comprimento médio de $3,2 \mathrm{~km}$, no campo cerrado nativo foram amostradas sete transeções, com comprimento médio de $2,54 \mathrm{~km}$, e na pastagem exótica cultivada foram percorridas seis transeções, com comprimento médio de $3,12 \mathrm{~km}$. Os levantamentos foram realizados entre 7:00h e 17:00h, por um único observador no período de seca, de maio a junho de 2010, sendo três a quatro transeções amostradas por dia. As transeções lineares foram percorridas repetidamente, porém em dias diferentes visando garantir independência entre as amostragens. Durante as amostragens foram registrados o número de detecções de indivíduos ou grupos de emas, bem como o número de indivíduos em cada grupo a distância perpendicular de cada grupo em relação 
à linha da transecção. Foi utilizado um aparelho de GPS para que o observador seguisse sobre a transeção linear, enquanto a percorria caminhando em uma velocidade de no máximo $3 \mathrm{~km} / \mathrm{h}$, e para verificar o ângulo formado entre os animais e a transeção linear. Um total de $438 \mathrm{~km}$ foi percorrido durante as amostragens. Os dados foram analisados através do programa Distace 6.0 (Buckland et al. 1993, Thomas et al. 2009, Thomas et al. 2010) e a seleção do modelo que melhor se ajusta aos dados observados foi baseada na minimização do Critério de Informação Akaike (Johnson \& Omland 2004). Foi aplicado também o teste não-paramétrico de Kruskal-Wallis para verificar se houve diferença nas taxas de encontro (número de detecções por quilômetro de transecto) nas três paisagens estudadas.

\section{RESULTADOS}

Ao longo de 438 quilômetros percorridos, houve 135 detecções de indivíduos ou grupos de $R$. americana. Nas três paisagens, o modelo que melhor se ajustou aos dados coletados foi half normal (Figura1). As maiores densidades de indivíduos e de grupos de $R$. americana foram encontradas no campo limpo nativo, enquanto que as menores no campo cerrado. As diferenças foram significativas entre campo cerrado e campo limpo, e entre campo cerrado e pastagem cultivada $(p<0,001)$. Entre campo limpo e pastagem cultivada não houve diferença significativa. Estimativas de densidade de grupos, número de indivíduos esperado por grupo, densidade de indivíduos e coeficientes de variação $(\mathrm{CV})$ estão representados na Tabela 1.
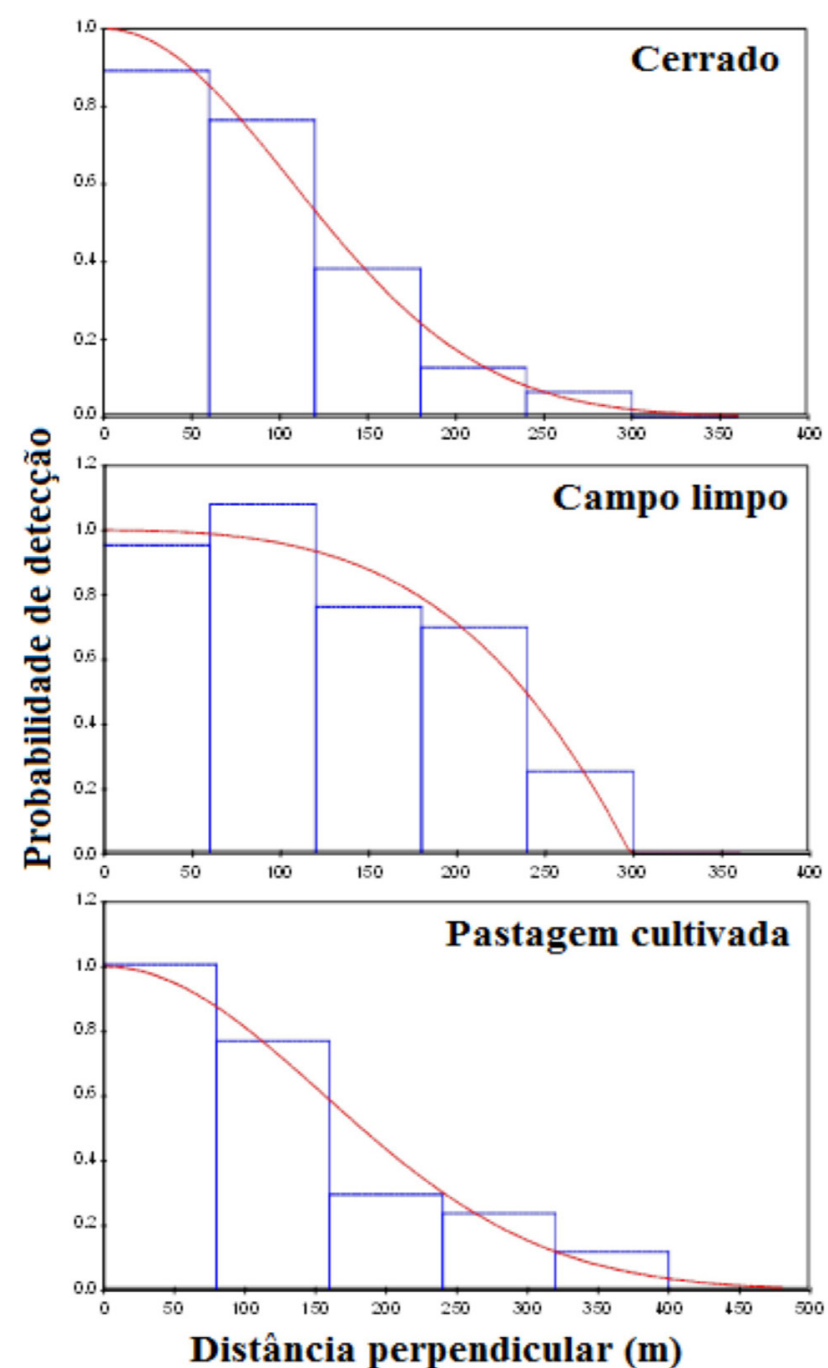

Figura 1: Curva de detectabilidade (linha) que melhor se ajustou aos grupos de emas (Rhea americana) observados (barras) a partir de transecções lineares conduzidas em diferentes paisagens na região da Nhecolândia, Pantanal, Brasil, de maio a junho de 2010.

Figure 1: Detection function (line) that best fit to the observed greater rhea (Rhea americana) groups (bar) from line transects surveyed in different landscapes of Nhecolândia region, Pantanal, Brazil, from May to June 2010. 
Tabela 1 Estimativas de densidade de grupos (DS), número de indivíduos esperado por grupo (E (S)), densidade de indivíduos (D) e coeficientes de variação (CV) utilizando o modelo "half-normal", em diferentes paisagens na região da Nhecolândia, Pantanal, Brasil, de maio a junho de 2010. Table 1: Estimated density of clusters (DS), expected cluster size (E(S)), individuals density (D) and coefficient of variation (CV) using half-normal model, at different landscapes of the Nhecolândia region, Pantanal, Brazil, from May to June 2010.

\begin{tabular}{|c|c|c|c|c|}
\hline Paisagem & $\begin{array}{c}\text { DS } \\
\left(\text { grupos } / \mathbf{k m}^{2} \text { ) }\right.\end{array}$ & $\begin{array}{c}\mathrm{E}(\mathrm{S}) \\
\text { (indivíduos/grupo) }\end{array}$ & $\begin{array}{c}\text { D } \\
\text { (indivíduos } / \mathbf{k m}^{2} \text { ) }\end{array}$ & $\begin{array}{c}\text { CV (D) } \\
(\%)\end{array}$ \\
\hline Cerrado & $0,651 \pm 0,130$ & $1,667 \pm 0,126$ & $1,086 \pm 0,232$ & 21.39 \\
\hline Campo limpo & $1,005 \pm 0,199$ & $2,071 \pm 0,152$ & $2,083 \pm 0,440$ & 21.16 \\
\hline Pastagem cultivada & $0,988 \pm 0,166$ & $2,055 \pm 0,232$ & $2,030 \pm 0,411$ & 20.27 \\
\hline
\end{tabular}

\section{DISCUSSÃO}

Os coeficientes de variação aqui encontrados estão de acordo com os cerca de $20 \%$ que Buckland et al. (1993) preconizam como aceitável para estimativas confiáveis de abundância. Isso confirma a possibilidade do uso do método da amostragem de distâncias para estimativas populacionais de $R$. americana sugerida por Desbiez \& Tomás (2003) e por Bellis et al. (2008).

Dados os aspectos comportamentais que $R$. americana apresenta na época reprodutiva, como por exemplo a formação de haréns, que no centrooeste do Brasil acontece de julho a setembro (Sick 1997), é importante reforçar a questão das amostragens terem sido realizadas no período não reprodutivo. Além disso, também fatores como a estação do ano e a estrutura do habitat influenciam o comportamento da espécie (Azevedo et al. 2010), o que provavelmente reflete em variações espaciais e temporais no tamanho dos grupos e e sua distribuição, mas é provável que isso não se reflita em variações importantes da abundância da espécie em escala de paisagem, já que emas não são conhecidas como migrantes ou nômades tróficas.

Houve variação na densidade de grupos e de indivíduos entre as três áreas, sendo que na paisagem com predomínio de cerrado e campo cerrado foi encontrada a menor densidade de $R$. americana. As maiores densidades obtidas na paisagem com predomínio de campo limpo nativo e naquela de pastagem cultivada indicam maior presença da espécie em fisionomias de estrutura com vegetação baixa, confirmando a afinidade de R. americana por áreas de campos ou pastagens, nativos ou exóticos, citada por Bellis et al. (2004), Azevedo et al. (2010) e Giordano et al. (2010). Provavelmente isso é resultado do balanço entre o ganho energético do forrageamento e os custos da vigilância em relação a predadores, no qual pode ser energeticamente mais vantajoso forragear em uma área desobstruída, em bandos mais numerosos (Reboreda \& Fernandez 1997, Fernandez et al. 2003, Carro \& Fernandez 2009). Além disso, é nessas áreas sem a presença de arbustos maiores, arvoretas ou árvores que $R$. americana realiza sua estratégia de fuga com maior sucesso, correndo em altas velocidades e desenhando um traçado sinuoso (Sick 1997, Bellis 2004, Azevedo et al. 2010), o que seria dificultado em áreas mais arborizadas ou arbustiva densa.

Já a indiferença em relação à alteração substancial na composição florística dos habitats abertos de pastagem cultivada aqui encontrada pode ser explicada pela característica onívora e generalista da espécie (Sick 1997). Azevedo et al. (2006), estudando a dieta de $R$. americana, identificaram itens como peixes, frutos, capim exótico braquiarão (Urochloa brizantha) e, em maioria, insetos. Dessa forma a ema é capaz de encontrar também nas pastagens cultivadas os recursos alimentares necessários (Azevedo et al. 2010), podendo inclusive aumentar suas densidades nestas áreas implantadas através da substituição de formação florestal ou de savana arborizada. 
Utilizando contagens aéreas, Hasenclever et al. (2004) estimaram a densidade de emas em 0,05 grupos $/ \mathrm{km}^{2}$ no Pantanal. Como os próprios autores concluíram, sobrevôo não é o método mais indicado para a contagem de emas, já que elas ocupam também áreas de cerrado, onde a detectabilidade é menor, e sugeriram que essa estimativa deve ser considerada como densidade mínima encontrada no Pantanal.

Investigando a relação entre pastagens cultivadas e campos naturais e diferentes espécies de aves campestres no Uruguai, Azpiroz \& Blake (2009) encontraram maiores densidades de $R$. americana na vegetação nativa do que na cultivada, porém os autores citam a caça nas áreas cultivadas avaliadas como um fator que poderia estar afetando os resultados do estudo. Já Bellis et al. (2004) encontraram mais indivíduos em pastagens cultivadas do que nas nativas. Giordano et al. (2008) encontraram maiores densidades de ema em campos e pastagens quando comparadas a áreas de agricultura e citam o recorrente estresse gerado pela atividade agrícola como causador de forte impacto negativo sobre a espécie. Esses resultados estão em concordância com os aqui documentados, uma vez que no Pantanal da Nhecolândia a espécie também apresentou maior densidade nos campos limpos, indiferentemente da sua composição florística. As densidades de $R$. americana aqui registradas foram maiores que as documentadas por todos autores acima citados. Possivelmente esse fato se deve às práticas de uso do solo tradicionalmente utilizadas no Pantanal de Nhecolândia, onde agricultura praticamente inexiste e a pecuária é de manejo extensivo. Além disso, nessa região a caça já deixou de representar ameaça a grande parte da fauna silvestre (Harris et al. 2005), sendo atualmente voltada quase que exclusivamente aos porcos asselvajados Sus scrofa, localmente conhecidos como porcos monteiros (Desbiez et al. 2011).

Estudos com outras espécies ou assembléias de aves indicam que as respostas à alteração de habitats campestres podem ser muito variadas dependendo tanto da espécie como também da região ou das novas características do local (Winter et al. 2005, Peterjohn 2003, Azpiroz \& Blake 2009). Apesar da descaracterização dos habitats ser citada como grande ameaça à ema, no Pantanal da Nhecolândia, a substituição da vegetação natural por pastagens de
Urochloa sp. parecem não prejudicar as populações de $R$. americana, podendo inclusive beneficiá-las através da criação de habitats extensos e estruturalmente mais favoráveis. Entretanto, outras espécies da fauna local não respondem da mesma maneira (p.e., Tizianel 2008, Antunes 2009, Mozerle 2011, Silveira 2011, Tomas et al. 2012). No contexto mais amplo, a manutenção da diversidade biológica precisa levar em conta os efeitos em comunidades e não apenas em espécies isoladas, já que a alteração na composição e estrutura de habitats naturais pode não afetar a riqueza de espécies no local, mas alterar profundamente a composição das comunidades, como tem sido observado no Pantanal em estudos envolvendo aves (Tizianel 2008), morcegos (Silveira 2011) e pequenos mamíferos (Mozerle 2011, Antunes 2009). Desta forma, o manejo das paisagens naturais no Pantanal precisa ser embasado em abordagens mais integradas e abrangentes, uma vez que sua alteração em larga escala pode ter efeitos negativos na conservação da biodiversidade, inclusive a diversidade funcional.

AGRADECIMENTOS: Este estudo contou com o apoio financeiro e logístico da Embrapa Pantanal pelo projeto SEG 02.10.06.007.00.00 SEG 02.07.50.003-02. Agradecemos à CAPES pela bolsa concedida e aos proprietários Heitor Miraglia Herrera e João Guilherme Monteiro por terem permitido a realização dos levantamentos em suas fazendas.

\section{REFERÊNCIAS}

ALHO, C.J.R. \& LACHER, T.E.Jr. 1991. Mammalian conservation in the Pantanal of Brazil. In: M. Mares \& D.J. Schmidly (eds). Latin American Mammalogy: History, Biodiversity and Conservation. University of Oklahoma Press, Norman, OK. 280-294.

ALHO, C.J.R. \& SABINO, J. 2011. A conservation agenda for the Pantanal's biodiversity. Brazilian Journal of Biology, São Carlos, v. 71 , n. 1 , supl. 1 .

ALMEIDA, T.I.R.; PARANHOS FILHO, A.C.; ROCHA, M.M.; SOUZA, G.F.; SÍGOLO, J.B. \& BERTOLO, R.A. 2009. Estudo sobre as diferenças de altimetria do nível da água de lagoas salinas e hipossalinas no Pantanal da Nhecolândia: um indicativo de funcionamento do mega sistema lacustre. Geociências 28 (4): 401-415.

ANTUNES, P.C. 2009. Uso de habitat e partição do espaço entre três espécies de pequenos mamíferos simpátricos no Pantanal Sulmato-grossense, Brasil. Dissertação de Mestrado. Universidade Federal de Mato Grosso do Sul, Campo Grande, MS, Brasil. 
AZEVEDO, C.S.; TINOCO, H.P.; FERRAZ, J.B. \& YOUNG, R.J. 2006. The fishing rhea: a new food item in the diet of wild greater rheas (Rhea americana, Rheidae, Aves). Revista Brasileira de Ornitologia, 14 (3): 285-287.

AZEVEDO, C.S.; FERRAZ, J.B.; TINOCO, H.P.; YOUNG, R.J. \& RODRIGUES, M. 2010. Time-activity budget of greater rheas (Rhea americana, Aves) on a human-disturbed area: the role of habitat, time of the day, season and group size. Acta Ethoologica, 13: 109-117. http://dx.doi.org/10.1007/s10211-010-0080-7

AZPIROZ, A.B. \& BLAKE, J.G. 2009. Avian assemblages in altered and natural grasslands in the northern campos of Uruguay. Condor, 111:21-35. http://dx.doi.org/10.1525/cond.2009.080111

BELLIS, L.M.; MARTELLA, M.B. \& NAVARRO, J.L. 2004. Habitat use by wild and captive-reared greater rheas Rhea americana in agricultural landscapes in Argentina. Oryx, 38: 304310. http://dx.doi.org/10.1017/S0030605304000547

BELLIS, L.M.; PIDGEON, A.M.; RADELOFF, V.C.; STLOUIS, V.; NAVARRO, J.L. \& MARTELLA, M.B. 2008. Modeling habitat suitability for greater rheas based on satellite image texture. Ecological Applications, 18:1956-1966. http:// dx.doi.org/10.1890/07-0243.1

BIRDLIFE INTERNATIONAL, 2012. Species factsheet: Rhea americana. Downloaded from http://www.birdlife.org (acessado em 03/04/2012).

BLAKE, E.R. 1977. A manual of Neotropical birds. University Chicago Press, Chicago Press, Chicago, Illinois. 674pp.

BOND, W.J. \& PARR, C.L. 2010. Beyond the forest edge: ecology, diversity and conservation of the grassy biomes. Biological Conservation, 143:2395-2404.http://dx.doi.org/10.1016/j. biocon.2009.12.012

BRENNAN, L.A. \& KUVLESKY JR, W.P. 2005. North American grassland birds: an unfolding conservation crisis? Journal of Wildlife Management, 69:1-13. http://dx.doi.org/10.2193/0022541X(2005)069<0001:NAGBAU>2.0.CO;2

BUCKLAND, S.T.; ANDERSON, D.R.; BURNHAM, K.P. \& LAAKE, J.L. 1993. Distance sampling: estimating abundance of biological populations. London: Chapman \& Hall. 446pp.

BUCKLAND, S.T.; ANDERSON, D.R.; BURNHAN, K.P.; LAAKE, J.L.; BORCHERS, D.L. \& THOMAS, L. 2001. Introduction to distance sampling: estimating abundance of biological populations. Oxford University Press. 432pp.

CARRO, M.E. \& FERNÁNDEZ, G.J. 2009. Scanning pattern of Greater Rheas, Rhea americana: collective vigilance would increase the probability of detecting a predator. Journal of Ethology, 27: 429-436. http://dx.doi.org/10.1007/s10164-0080137-5

CASSEY, P. 1999. Estimating animal abundance by distance sampling techniques. Conservation Advisory Science Noies No. 237, Department of Conservation, Wellington. 12pp.

CONROY, M.J. \& CARROL, J.P. 2009. Distance sampling for estimating density and abundance. Pp. 115-134. In: M.J. Conroy \& J.P. Carrol (eds.). Quantitative Conservation of Vertebrates. Wiley-Blackwell, Hoboken, NJ. 342p.

DESBIEZ, A. \& TOMÁS, W.M. 2003. Aplicabilidade do método de amostragem de distâncias em levantamentos de médios e grandes vertebrados no Pantanal. Boletim de Pesquisa e Desenvolvimento, EMBRAPA.53: 5-16.

DESBIEZ, A.L.J.; PIOVEZAN, A.K.U. \& BODMER, R.E. 2011. Invasive species and bushmeat hunting contributing to wildlife conservation: the case of feral pigs in a Neotropical wetland. Oryx, 45(1): 78-83. http://dx.doi.org/10.1017/S0030605310001304

ERIZE, F.; MATA, J.R.R. \& RUMBOLL, M. 2006. Birds of South America. Non-Passerines: rheas to woodpeckers. Princeton University Press, Oxford, $384 \mathrm{p}$.

FERNANDEZ, G.J.; CAPURRO, A.F. \& REBOREDA, J.C. 2003. Effect of group size on individual and collective vigilance in greater rheas. Ethology, 109: 413-425. http://dx.doi.org/10.1046/ j.1439-0310.2003.00887.x

GIORDANO, P.F.; BELLIS, L.M.; NAVARRO, J.L. \& MARTELLA, M.B. 2008. Abundance and spatial distribution of greater rheas Rhea americana in two sites of pampas grasslands with different land use. Bird Conservation International, 18: 6370. http://dx.doi.org/10.1017/S0959270908000075

GIORDANO, P.F.; NAVARRO, J.L. \& MARTELLA, M.B. 2010. Building large-scale spatially explicit models to predict the distribution of suitable habitat patches for the Greater rhea (Rhea americana), a near-threatened species. Biological Conservation, 143: 357-365. http://dx.doi.org/10.1016/j.biocon.2009.10.022

HARRIS, M.B.; TOMÁS, W.M.; MOURÃO, G.; DA SILVA, C.J.; GUIMARÃES, E.; SONODA, F. \& FACHIM, E. 2005. Safeguarding the Pantanal wetlands: threats and conservation initiatives. Conservation Biology 19: 714-720. http://dx.doi. org/10.1111/j.1523-1739.2005.00708.x

HASENCLEVER, L.; REIMAN， C.; MOURÃO, G.M. \& CAMPOS, Z.M.S. 2004. Densidades, tamanho de grupo e reprodução de emas no Pantanal sul. Boletim de Pesquisa e Desenvolvimento, Embrapa Pantanal, 55: 1-19. 
JOHNSON, J.B. \& OMLAND, K.S. 2004. Model selection in ecology and evolution. Trends in Ecology and Evolution, 19: 101108. http://dx.doi.org/10.1016/j.tree.2003.10.013

JUNK,W.J. \& CUNHA, C.N. 2005. Pantanal: A large South American wetland at a crossroads. Ecological Engineering, 24: 391-401. http://dx.doi.org/10.1016/j.ecoleng.2004.11.012

JUNK, W.J.; CUNHA, C.N.; KNOPF, F.L. 1994. Avian assemblages in altered grasslands. Studies in Avian Biology, 15:247-257.

MARTIN, T.E. \& FINCH, D.M. 1995. Ecology and management of neotropical migratory birds. Oxford University Press, New York. 489 p.

MERCANTE, M.A.; RODRIGUES, S.C. \& ROSS, J.L.S. 2011. Geomorphology and habitat diversity in the Pantanal. Brazilian Journal of Biology, 71: 233-240.

MOZERLE, H.B. 2011. Probabilidade de ocupação de pequenos mamíferos em uma região do Pantanal, Mato Grosso do Sul, Brasil. Dissertação de Mestrado. Universidade Federal de Mato Grosso do Sul, Campo Grande, MS, Brasil.

MURPHY, M.T. 2003. Avian population trends within the evolving agricultural landscape of eastern and central United States. The Auk, 120:20-34. http://dx.doi.org/10.2307/4090137

PADOVANI, C.R.; CRUZ, M.L.L. \& PADOVANI, S.L.A.G. 2004. Desmatamento do Pantanal brasileiro para o ano 2000. In: IV Simposio Sobre Recursos Naturais e Socio-Economicos do Pantanal, Embrapa Pantanal. Corumba, Brasil.

PETERJOHN, B.G. 2003. Agricultural landscapes: Can they support healthy bird populations as well as farm products? The Auk, 120: 14-19. http://dx.doi.org/10.2307/4090136

POTT, A. 1982. Pastagens das sub-regiões dos Paiaguás e da Nhecolândia do Pantanal Mato-grossense. Corumbá, EMBRAPA, UEPAE de Corumbá. Circular Técnica, 10: 49p.

POTT, V.J.; POTT, A.; RATTER, J.A. \& VALLS, J.M.F. 1986. Flora da fazenda Nhumirim, Nhecolândia, Pantanal. Relação preliminar. Corumbá, EMBRAPA/CPAP (Pesquisa em Andamento, 5).

REBOREDA, J.C. \& FERNANDEZ, G. J. 1997. Sexual, seasonal and group size differences in the allocation of time between vigilance and feeding in the greater rhea, Rhea americana. Ethology, 103: 198-207. http://dx.doi. org/10.1111/j.1439-0310.1997.tb00116.x

SANTOS, S.A.; COSTA, C.; SOUZA, G.S.E.; MORAES, A.S. \& ARRIGONI, M.D.B. 2002. Qualidade da dieta selecionada por bovinos na sub-região da Nhecolândia, Pantanal. Revista
Brasileira de Zootecnia, 31: 663-673. http://dx.doi.org/10.1590/ S1516-35982002000700008

SEIDL, A.F.; DE SILVA, J.S.V. \& MORAES, A.S. 2001. Cattle ranching and deforestation in the Brazilian Pantanal. Ecological Economics, 36:413-425.http://dx.doi.org/10.1016/S09218009(00)00238-X

SICK, H. 1997. Ornitologia brasileira. Rio de Janeiro, Ed. Nova Fronteira, $862 \mathrm{p}$

SILVEIRA, L.F. \& STRAUBE, F.C. 2008. Aves ameaçadas de extinção no Brasil. In: A.B.M. Machado, G.M. Drumond \& A.P. Paglia (eds.) Livro vermelho da fauna brasileira ameaçada de extinção. Brasília: Ministério do Meio Ambiente.

SILVEIRA, M. 2011. Influência da estrutura da vegetação em morcegos (Mammalia, Chiroptera) no Pantanal da Nhecolândia, Brasil. Dissertação e Mestrado. Universidade Federal de Mato Grosso do Sul, Campo Grande, MS, Brasil.

THOMAS, L.; LAAKE, J.L.; REXSTAD, E.; STRINDBERG, S.; MARQUES, F.F.C.; BUCKLAND, S.T.; BORCHERS, D.L.; ANDERSON, D.R.; BURNHAM, K.P.; BURT, M.L.; HEDLEY, S.L.; POLLARD, J.H.; BISHOP, J.R.B. \& MARQUES, T.A. 2009. Distance 6.0. Release 2. Research Unit for Wildlife Population Assessment, University of St. Andrews, UK. http:// www.ruwpa.st-and.ac.uk/distance/.

THOMAS, L.; BUCKLAND, ST.; REXSTAD, E.A.; LAAKE, J.L.; STRINDBERG, S.; HEDLEY, S.L.; BISHOP, J.R.B.; MARQUES, T.A. \& BURNHAM, K.P. 2010. Distance software: design and analysis of distance sampling surveys for estimating population size. Journal of Applied Ecology, 47: 5-14. http:// dx.doi.org/10.1111/j.1365-2664.2009.01737.x

THOMPSON, W.L.; WHITE, G.C. \& GOWAN, C. 1998. Monitoring Vertebrate Populations. Academic Press, London.

TIZIANEL, F.A.T. 2008. Efeito da complexidade da vegetação de fitofisionomias naturais e pastagens cultivadas sobre a comunidade de aves em duas fazendas no Pantanal da Nhecolandia, Corumbá, Mato Grosso do Sul. Dissertação de Mestrado. Universidade Federal do Mato Grosso do Sul, Campo Grande, MS, Brasil.

TOMAS, M.A.; TOMAS, W.M. \& RODRIGUES, F.H.G. 2012.. Densidade e uso de recursos por veado-campeiro (Ozotoceros bezoarticus) em tres paisagens diferentes no Pantanal, MS. Oecologia Australis, 16(4): 914-932. http://dx.doi.org/10.4257/ oeco.2012.1604.14

TOMÁS, W.M.; SOUZA L.L. \& TUBELIS, D.P. 2004. Espécies de aves ameaçadas que ocorrem no Pantanal. In: IV Simposio 
Sobre Recursos Naturais e Socio-Economicos do Pantanal, Embrapa Pantanal. Corumbá, Brasil.

VICKERY, P.D.; TUBARO, P.L.; CARDOSO DA SILVA, J.M.; PETERJOHN, B.G.; HERKERT J.R. \& CAVALCANTI, R.B. 1999. Conservation of grassland birds in the western hemisphere. Studies in Avian Biology, 19:2-26.

VEECH, J.A. 2006. A comparison of landscapes occupied by increasing and decreasing populations of grassland birds. Conservation Biology 20:1422-1432. http://dx.doi.org/10.1111/ j.1523-1739.2006.00487.x

WANTZEN, K.M.; PETERMANN, P.; STRUSSMANN, C.; MARQUES, M.I. \& ADIS, J. 2006. Biodiversity and its conservation in the Pantanal of Mato Grosso, Brazil. Aquatic Sciences, 68:278-309.http://dx.doi.org/10.1007/s00027-0060851-4

WINTER, M.; JOHNSON, D.H. \& SHAFFER, J.A. 2005. Variability in vegetation effects on density and nesting success of grassland birds. Journal of Wildlife Management, 69: 185-197. http://dx.doi.org/10.2193/0022-541X(2005)069<0185:VIVEOD $>2.0 . \mathrm{CO} ; 2$

Submetido em 05/05/2012 Aceito em 20/12/2012 